\title{
The generalized Catalan equation in positive characteristic
}

\author{
Peter Koymans \\ p.h.koymans@math.leidenuniv.nl
}

May 26, 2021

In this article we will bound $m$ and $n$ for the generalized Catalan equation in characteristic $p>0$.

\section{Notation}

Let $K=\mathbb{F}_{p}\left(z_{1}, \ldots, z_{r}\right)$ be a finitely generated field over $\mathbb{F}_{p}$. Our main result in this article is as follows.

Theorem 1. Let $a, b \in K^{*}$ be given. Consider the equation

$$
a x^{m}+b y^{n}=1
$$

in $x, y \in K$ and integers $m, n>1$ coprime with $p$ satisfying

$$
(m, n) \notin\{(2,2),(2,3),(3,2),(2,4),(4,2),(3,3)\} .
$$

Then there is a finite set $\mathcal{T} \subseteq K^{2}$ such that for any solution $(x, y, m, n)$ of (1), there is a $(\gamma, \delta) \in \mathcal{T}$ and $t \in \mathbb{Z}_{\geq 0}$ such that

$$
a x^{m}=\gamma^{p^{t}}, b y^{n}=\delta^{p^{t}}
$$

\section{Discussion of Theorem 1}

In the case $a=b=1$, a stronger and effective result was proven in [6] based on the work of [1].

Let us now show that the conditions on $m$ and $n$ are necessary. If (2) fails, then (11) defines a curve of genus 0 or 1 over $K$. It is clear that (3) can fail in this case. It is also essential that $m$ and $n$ are coprime with $p$. Take for example $a=b=1$. Then any solution of

$$
x+y=1
$$

with $x, y \in K$ and $x, y \notin \overline{\mathbb{F}_{p}}$ gives infinitely many solutions of the form (3) after applying Frobenius.

The generalized Catalan equation over function fields was already analyzed in [8], where the main theorem claims that the generalized Catalan equation has no solutions for $m$ and $n$ sufficiently large. Unfortunately, it is not hard to produce counterexamples to the main theorem given there. Following the notation in [8], we choose $k=\mathbb{F}_{p}, K=k(u), a=x=u$, $b=y=1-u$ and $m=n=p^{t}-1$ for $t \in \mathbb{Z}_{\geq 0}$. Then we have

$$
a x^{m}+b y^{n}=u \cdot u^{p^{t}-1}+(1-u) \cdot(1-u)^{p^{t}-1}=1
$$


due to Frobenius, illustrating the need of (3).

\section{Proof of Theorem 1}

For our proof we will need a generalization of Mason's ABC-theorem for function fields in one variable to an arbitrary number of variables. Such a result is given in [2].

Proof. Let $(x, y, m, n)$ be an arbitrary solution. Without loss of generality we may assume that $\left\{z_{1}, \ldots, z_{k}\right\}$ forms a transcendence basis of $K / \mathbb{F}_{p}$.

We write $t:=r-k$ and rename $z_{k+1}, \ldots, z_{r}$ as $y_{1}, \ldots, y_{t}$ respectively. Define

$$
A_{0}:=\mathbb{F}_{p}\left[z_{1}, \ldots, z_{k}\right], \quad K_{0}:=\mathbb{F}_{p}\left(z_{1}, \ldots, z_{k}\right) .
$$

Then

$$
A=A_{0}\left[y_{1}, \ldots, y_{t}\right], \quad K=K_{0}\left(y_{1}, \ldots, y_{t}\right) .
$$

Note that $A$ and $K$ are respectively the coordinate ring and the function field of an affine algebraic variety $V$ over $\mathbb{F}_{p}$. Then the algebraic closure of $\mathbb{F}_{p}$ in $K$ is a finite extension of $\mathbb{F}_{p}$, say $\mathbb{F}_{q}$ with $q=p^{n}$ for some $n \in \mathbb{Z}_{>0}$. Because $\mathbb{F}_{q}$ is perfect, it follows that $K$ is separably generated over $\mathbb{F}_{q}$, see Theorem 26.2 in $[9]$.

Taking the projective closure of $V$ and normalizing gives a projective variety non-singular in codimension one, still with function field $K$. Let us call the variety thus obtained $W$. Our goal will be to introduce a height on $K$. For later purposes it will be useful to do this in a slightly more general setting. So let $X$ be a projective variety, non-singular in codimension one, defined over a perfect field $k$. We write $L$ for the function field of $X$ and we assume that $k$ is algebraically closed in $L$.

Fix a projective embedding of $X$ such that $X \subseteq \mathbb{P}_{k}^{M}$ for some positive integer $M$. Then a prime divisor $\mathfrak{p}$ of $X$ over $k$ is by definition an irreducible subvariety of codimension one. Recall that for a prime divisor $\mathfrak{p}$ the local ring $\mathcal{O}_{\mathfrak{p}}$ is a discrete valuation ring, since $X$ is non-singular in codimension one. Following [4] we will define heights on $X$. To do this, we start by defining a set of normalized discrete valuations

$$
M_{L}:=\left\{\operatorname{ord}_{\mathfrak{p}}: \mathfrak{p} \text { prime divisor of } X\right\},
$$

where $\operatorname{ord}_{\mathfrak{p}}$ is the normalized discrete valuation of $L$ corresponding to $\mathcal{O}_{\mathfrak{p}}$. If $v=\operatorname{ord}_{\mathfrak{p}} \in M_{L}$, we define for convenience $\operatorname{deg} v:=\operatorname{deg} \mathfrak{p}$ with $\operatorname{deg} \mathfrak{p}$ being the projective degree in $\mathbb{P}_{k}^{M}$. Then the set $M_{L}$ satisfies the sum formula for all $x \in L^{*}$

$$
\sum_{v} v(x) \operatorname{deg} v=0
$$

If $P$ is a point in $\mathbb{P}^{r}(L)$ with coordinates $\left(y_{0}: \ldots: y_{r}\right)$ in $L$, then its (logarithmic) height is

$$
h_{L}(P)=-\sum_{v} \min _{i}\left\{v\left(y_{i}\right)\right\} \operatorname{deg} v
$$

Furthermore we define for an element $x \in L$

$$
h_{L}(x)=h_{L}(1: x) .
$$

We will need the following properties of the height. 
Lemma 2. Let $x, y \in L$ and $n \in \mathbb{Z}$. The height defined by (4) has the following properties:

(a) $h_{L}(x)=0 \Leftrightarrow x \in k$;

(b) $h_{L}(x+y) \leq h_{L}(x)+h_{L}(y)$;

(c) $h_{L}(x y) \leq h_{L}(x)+h_{L}(y)$;

(d) $h_{L}\left(x^{n}\right)=n h_{L}(x)$;

(e) Suppose that $k$ is a finite field and let $C>0$ be given. Then there are only finitely many $x \in L^{*}$ satisfying $h_{L}(x) \leq C$;

(f) $h_{L}(x)=h_{\bar{k} \cdot L}(x)$.

Proof. Property (a) is Proposition 4 of [3] (p. 157), while properties (b), (c) and (d) are easily verified. Property (e) is proven in [5]. Finally, property (f) can be found after Proposition 3.2 in [4] (p. 63).

Let us now dispose with the case $a x^{m} \in \mathbb{F}_{q}$. Then

$$
2 h_{K}(x) \leq m h_{K}(x)=h_{K}\left(x^{m}\right) \leq h_{K}\left(a x^{m}\right)+h_{K}\left(a^{-1}\right)=h_{K}\left(a^{-1}\right),
$$

hence there are only finitely many possibilities for $x$. Now observe that $a x^{m} \in \mathbb{F}_{q}$ implies $b y^{n} \in \mathbb{F}_{q}$. By the same argument we get finitely many possibilities for $y$, so we are done in this case.

From now on we will assume $a x^{m} \notin \mathbb{F}_{q}$ and hence $b y^{n} \notin \mathbb{F}_{q}$. Then it follows that

$$
h_{K}\left(a x^{m}\right), h_{K}\left(b y^{n}\right) \neq 0 \text {. }
$$

Write

$$
a x^{m}=\gamma^{p^{t}}, b y^{n}=\delta^{p^{s}}
$$

for some $t, s \in \mathbb{Z}_{\geq 0}$ and $\gamma, \delta \notin K^{p}$. After substitution we get

$$
\gamma^{p^{t}}+\delta^{p^{s}}=1
$$

Extracting $p$-th roots gives $t=s$ and hence

$$
\gamma+\delta=1
$$

Our goal will be to apply the main theorem of [2] to (5). For completeness we repeat it here.

Theorem 3. Let $X$ be a projective variety over an algebraically closed field $k$ of characteristic $p>0$, which is non-singular in codimension one. Let $L=k(X)$ be its function field and let $M_{L}$ be as above. Let $L_{1}, \ldots, L_{q}, q \geq n+1$, be linear forms in $n+1$ variables over $k$ which are in general position. Let $\mathbf{X}=\left(x_{0}: \ldots: x_{n}\right) \in \mathbb{P}^{n}(L)$ be such that $x_{0}, \ldots, x_{n}$ are linearly independent over $K^{p^{m}}$ for some $m \in \mathbb{N}$. Then, for any fixed finite subset $S$ of $M_{L}$, the following inequality holds:

$$
\begin{aligned}
& (q-n-1) h\left(x_{0}: \ldots: x_{n}\right) \\
& \leq \sum_{i=1}^{q} \sum_{v \notin S} \operatorname{deg} v \min \left\{n p^{m-1}, v\left(L_{i}(\mathbf{X})\right)-\min _{0 \leq j \leq n}\left\{v\left(x_{j}\right)\right\}\right\} \\
& \quad+\frac{n(n+1)}{2} p^{m-1}\left(C_{X}+\sum_{v \in S} \operatorname{deg} v\right),
\end{aligned}
$$

where $C_{X}$ is a constant depending only on $X$. 
Proof. See Theorem in [2].

Note that Theorem 3 requires that the ground field $k$ is algebraically closed. But a constant field extension does not change the height by Lemma 2(f). Hence we can keep working with our field $K$ instead of $\overline{\mathbb{F}_{p}} \cdot K$. Define the following three linear forms in two variables $X, Y$

$$
\begin{aligned}
& L_{1}=X \\
& L_{2}=Y \\
& L_{3}=X+Y .
\end{aligned}
$$

We apply Theorem 3 with our $W$, the above $L_{1}, L_{2}, L_{3}$ and $\mathbf{X}=(\gamma: \delta) \in \mathbb{P}^{1}(K)$. We claim that $\gamma$ and $\delta$ are linearly independent over $K^{p}$. Suppose that there are $e, f \in K^{p}$ such that

$$
e \gamma+f \delta=0 .
$$

Together with $\gamma+\delta=1$ we find that

$$
0=e \gamma+f \delta=e(1-\delta)+f \delta=e+(f-e) \delta .
$$

If $e \neq f$, then this would imply that $\delta \in K^{p}$, contrary to our assumptions. Hence $e=f$, but then we find

$$
0=e \gamma+f \delta=e
$$

and we conclude that $e=f=0$ as desired.

We still have to choose the subset $S$ of $M_{K}$ to which we apply Theorem 3. First we need to make some preparations. From now on $v$ will be used to denote an element of $M_{K}$. Define

$$
\begin{aligned}
& N_{0}:=\{v: v(a) \neq 0 \vee v(b) \neq 0\} \\
& N_{1}:=\{v: v(a)=0, v(b)=0, v(\gamma)>0\} \\
& N_{2}:=\{v: v(a)=0, v(b)=0, v(\delta)>0\} \\
& N_{3}:=\{v: v(a)=v(b)=0, v(\gamma)=v(\delta)<0\} .
\end{aligned}
$$

It is clear that $N_{0}, N_{1}, N_{2}$ and $N_{3}$ are finite disjoint sets. Before we proceed, we make a simple but important observation in the form of a lemma.

Lemma 4. Let $(\gamma, \delta)$ be a solution of (5). If $v(\gamma)<0$ or $v(\delta)<0$, then

$$
v(\gamma)=v(\delta)<0
$$

Proof. Obvious.

Recall that

$$
h_{K}(\gamma)=\sum_{v} \max (0, v(\gamma)) \operatorname{deg} v=\sum_{v}-\min (0, v(\gamma)) \operatorname{deg} v
$$

and

$$
h_{K}(\delta)=\sum_{v} \max (0, v(\delta)) \operatorname{deg} v=\sum_{v}-\min (0, v(\delta)) \operatorname{deg} v
$$


Lemma 4 tells us that

$$
\sum_{v}-\min (0, v(\gamma)) \operatorname{deg} v=\sum_{v}-\min (0, v(\delta)) \operatorname{deg} v,
$$

hence

$$
\begin{aligned}
h_{K}(\gamma)=h_{K}(\delta) & =\sum_{v} \max (0, v(\gamma)) \operatorname{deg} v=\sum_{v}-\min (0, v(\gamma)) \operatorname{deg} v \\
& =\sum_{v} \max (0, v(\delta)) \operatorname{deg} v=\sum_{v}-\min (0, v(\delta)) \operatorname{deg} v
\end{aligned}
$$

We will use these different expressions for the height throughout. Let us now derive elegant upper bounds for $N_{1}, N_{2}$ and $N_{3}$. Again we will phrase it as a lemma.

Lemma 5. Let $(\gamma, \delta)$ be a solution of (5). Then

$$
\begin{aligned}
& h_{K}(\gamma)=h_{K}(\delta) \geq m \sum_{v \in N_{1}} \operatorname{deg} v, \\
& h_{K}(\gamma)=h_{K}(\delta) \geq n \sum_{v \in N_{2}} \operatorname{deg} v, \\
& h_{K}(\gamma)=h_{K}(\delta) \geq \operatorname{lcm}(m, n) \sum_{v \in N_{3}} \operatorname{deg} v .
\end{aligned}
$$

Proof. We know that

$$
h_{K}(\gamma)=h_{K}(\delta)=\sum_{v} \max (0, v(\gamma)) \operatorname{deg} v \geq \sum_{v \in N_{1}} \max (0, v(\gamma)) \operatorname{deg} v
$$

Now let $v \in N_{1}$. This means that $v(a)=v(b)=0$ and $v(\gamma)>0$. Then $a x^{m}=\gamma^{p^{t}}$ implies

$$
v(a)+m v(x)=p^{t} v(\gamma)
$$

and hence $m v(x)=p^{t} v(\gamma)$. But $m$ and $p$ are coprime by assumption, so we obtain $m \mid v(\gamma)$. Because $v(\gamma)>0$, this gives $v(\gamma) \geq m$ and we conclude that

$$
h_{K}(\gamma)=h_{K}(\delta) \geq m \sum_{v \in N_{1}} \operatorname{deg} v
$$

Using

$$
h_{K}(\gamma)=h_{K}(\delta)=\sum_{v} \max (0, v(\delta)) \operatorname{deg} v \geq \sum_{v \in N_{2}} \max (0, v(\delta)) \operatorname{deg} v
$$

we find in a similar way that

$$
h_{K}(\gamma)=h_{K}(\delta) \geq n \sum_{v \in N_{2}} \operatorname{deg} v
$$

It remains to be proven that

$$
h_{K}(\gamma)=h_{K}(\delta) \geq \operatorname{lcm}(m, n) \sum_{v \in N_{3}} \operatorname{deg} v .
$$


Now we use

$$
\begin{aligned}
h_{K}(\gamma)=h_{K}(\delta) & =\sum_{v}-\min (0, v(\gamma)) \operatorname{deg} v=\sum_{v}-\min (0, v(\delta)) \operatorname{deg} v \\
& \geq \sum_{v \in N_{3}}-\min (0, v(\gamma)) \operatorname{deg} v=\sum_{v \in N_{3}}-\min (0, v(\delta)) \operatorname{deg} v .
\end{aligned}
$$

Now take $v \in N_{3}$. Then $v(\gamma)=v(\delta)<0$. In the same way as before, we can show that $m \mid v(\gamma)$ and $n \mid v(\delta)$. But $v(\gamma)=v(\delta)<0$ by Lemma 4, so we find that

$$
h_{K}(\gamma)=h_{K}(\delta) \geq \operatorname{lcm}(m, n) \sum_{v \in N_{3}} \operatorname{deg} v
$$

as desired.

Define

$$
S:=N_{0} \cup N_{1} \cup N_{2} \cup N_{3} .
$$

Suppose that $v \notin S$. We claim that

$$
v(\gamma)=v(\delta)=0
$$

But $v \notin S$ implies $v \notin N_{0}$, so certainly $v(a)=v(b)=0$. Furthermore, we have that $v \notin N_{1}$ and $v \notin N_{2}$, which means that $v(\gamma) \leq 0$ and $v(\delta) \leq 0$. If $v(\gamma)<0$ or $v(\delta)<0$, then Lemma 4 gives $v \in N_{3}$, contradicting our assumption $v \notin S$. Hence $v(\gamma)=v(\delta)=0$ as desired.

From our claim it follows that we have for $v \notin S$ and $i=1,2,3$

$$
v\left(L_{i}(\gamma, \delta)\right)=\min (v(\gamma), v(\delta)) .
$$

Theorem 3 tells us that

$$
h_{K}(\gamma: \delta) \leq C_{W}+\sum_{v \in S} \operatorname{deg} v,
$$

where $C_{W}$ is a constant depending on $W$ only. By Lemma 5 we find that

$$
\begin{aligned}
\sum_{v \in S} \operatorname{deg} v & =\sum_{v \in N_{0}} \operatorname{deg} v+\sum_{v \in N_{1}} \operatorname{deg} v+\sum_{v \in N_{2}} \operatorname{deg} v+\sum_{v \in N_{3}} \operatorname{deg} v \\
& \leq C_{a, b}+\left(\frac{1}{m}+\frac{1}{n}+\frac{1}{\operatorname{lcm}(m, n)}\right) h_{K}(\gamma)
\end{aligned}
$$

where $C_{a, b}$ is a constant depending on $a$ and $b$ only. Now (2) implies

$$
\frac{1}{m}+\frac{1}{n}+\frac{1}{\operatorname{lcm}(m, n)}<0.9
$$

hence

$$
h_{K}(\gamma: \delta) \leq 10\left(C_{W}+C_{a, b}\right)
$$

But $\gamma+\delta=1$ gives

$$
h_{K}(\gamma)=h_{K}(\delta)=h_{K}(\gamma: \delta) .
$$

The theorem now follows from Lemma 2 (e). 


\section{Discussion of Theorem 1}

The conclusion of Theorem 1 tells us that there is a finite set $\mathcal{T} \subseteq K^{2}$ such that for any solution $(x, y, m, n)$ of (1), there is a $(\gamma, \delta) \in \mathcal{T}$ and $t \in \mathbb{Z}_{\geq 0}$ such that

$$
a x^{m}=\gamma^{p^{t}}, b y^{n}=\delta^{p^{t}} .
$$

Since $\mathcal{T}$ is finite, we may assume that $\gamma$ and $\delta$ are fixed in the above two equations. The resulting equation is studied in [7].

\section{References}

[1] B. Brindza, The Catalan equation over finitely generated integral domains, Publ. Math. Debrecen 42 (1993), 193-198.

[2] L.-C. Hsia, J.T.-Y. Wang, The ABC theorem for higher-dimensional function fields, Trans. Amer. Math. Soc. 356 (2004), 2871-2887.

[3] S. Lang, Introduction to Algebraic Geometry, Addison-Wesley, Redwood City, CA, 1973.

[4] S. Lang, Fundamentals of Diophantine Geometry, Springer, Berlin, 1983.

[5] D.W. Masser, Mixing and Linear Equations over Groups in positive characteristic, Isreal Journal of Mathematics 142 (2004), 189-204.

[6] P. Koymans, The Catalan equation, http://pub.math.leidenuniv.nl/ koymansph/MasterThesis.pdf

[7] P. Koymans, Power Equation, http://pub.math.leidenuniv.nl/ koymansph/PowerEquation.pdf

[8] J.H. Silverman, The Catalan equation over function fields, Trans. Amer. Math. Soc. 273 (1982), 201-205.

[9] H. Matsumura, Commutative ring theory, Cambridge University Press, Cambridge, UK, 1986. 